\title{
Comparison of Heart Rate Variability Assessment During Exercise from Polar RS800 and ECG
}

\author{
David Hernando ${ }^{1,2}$, Nuria Garatachea ${ }^{3,4}$, Jose A. Casajús ${ }^{3,4}$, Raquel Bailón ${ }^{1,2}$ \\ ${ }^{1}$ BSICoS Group, I3A, IIS Aragón, University of Zaragoza, Spain \\ ${ }^{2}$ CIBER - Bioingeniería, Biomateriales y Nanomedicina (CIBER-BBN), Spain \\ ${ }^{3}$ GENUD Research Group, Faculty of Health and Sport Sciences, IA2, IIS Aragón, University of \\ Zaragoza, Spain \\ ${ }^{4}$ CIBER - Fisiopatología de la Obesidad y Nutrición (CIBERObn), Spain
}

\begin{abstract}
There are several mobile and easy to use heart rate monitors which which allow heart rate variability (HRV) analysis during exercise, but there is a need to validate them during exercise of high intensities. A previous study revealed that mean heart rate and low frequency $(L F)$ power measurements were interchangeable between Polar RS800 and ECG, but instantaneous high frequency $(H F)$ power presented low agreement $(A)$ during high intensities of exercise $(A<0.7)$. In this study we want to analyze if, even with that disagreement in HF power, the same conclusions can be extracted regarding the response of ANS to exercise from Polar HRV measurements compared to those extracted from ECG.

23 male volunteers performed an exercise stress test on a cycle ergometer while wearing a Polar RS800 device and ECG being recorded simultaneously. A time-frequency spectral analysis was performed to extract the instantaneous mean heart rate and $L F$ and $H F$ powers, the latter guided by respiration. Spectral components related to cardiolocomotor coupling (CC) were identified and corrected when overlapping with other components. Oxygen consumption information was used to establish 4 different intervals of different exercise intensity.

No significant differences between ECG and Polar HRV measurements were found in any interval. Both showed an increase in normalized LF power in low intensities and a significant decrease in medium to high intensities of exercise, with an opposite behaviour in the case of normalized HF. CC components reached about $20 \%$ of the total power in the last intervals, with no significant differences between ECG and Polar.
\end{abstract}

\section{Introduction}

Heart rate variability (HRV) remains a powerful source for autonomic nervous system (ANS) assessment by using simple and non-invasive techniques. It is used in both clinical and research environments and over a broad spectrum of disciplines concerned with autonomic control of the heart ranging from cardiology to psychology. Advances in technology have allowed the development of mobile and easy to use heart rate monitors that allow the quantification of inter-beat intervals (RR intervals) and thus the analysis of heart rate variability. Commercial devices (like Polar, Garmin, Tomtom, or Suunto heart rate monitors, among others) appear as a cheaper alternative, usually providing software which allows for an affordable and a user-friendly method to determine short-term HRV outside of the laboratory setting.

These heart rate monitors have been used by scientists for HRV analysis in sport sciences, medicine and other fields of research [1-3]. Several studies have validated these devices against different ECG systems, showing promising results at rest [4-6]. However, there are very few studies that validate HRV measurements from heart rate monitor devices during dynamic exercise of high intensity, which is characterized by a higher level of noise than in static exercise, larger variations in mean heart rate (HR) and respiratory frequency and the appearance of cardiolocomotor coupling (CC) components during high intensities.

In a previous study [12], we evaluated the agreement and reliability between the HRV analysis derived from the RR series recorded by the HR monitor Polar RS800 and HRV analysis derived from a simultaneous ECG during dynamic exercise of low, medium and high intensity. At rest, Polar measurements showed high agreement and reliability indices as expected. During high intensities of exercise, however, high frequency (HF) parameters showed low agreement.

The aim of this study is to evaluate changes in ANS during high intensity exercise using Polar measurements, despite the low agreement in HF parameters, to see whether the same information can be extracted than from measure- 
ments derived directly from the ECG.

\section{Methods and materials}

\subsection{Database}

The database, described in [12], consists on ECG and Polar recordings from 23 healthy volunteers who regularly participate in sports activities. The study protocol was approved by the institutional ethics committee and was in accordance with the Declaration of Helsinki for Human Research of 1974 (last modified in 2008). Written informed consent was obtained from each subject.

All subjects completed a submaximal test on a cycle ergometer. The test was divided into three different phases: resting, exercise and recovery phase. During the resting phase, the subjects were continuously monitored while seated at rest for $5 \mathrm{~min}$. The exercise phase started on the cycle ergometer at $75 \mathrm{~W}$ work load, increasing at a rate of $25 \mathrm{Wmin}-1$. The cadence frequency was fixed at $80 \mathrm{rpm}$. This phase lasted until the subject reached his $90 \%$ maximum heart rate, which was determined in previous tests by a physician. Then, the work load was kept constant for two more minutes. The recovery phase consisted of $5 \mathrm{~min}$ of pedalling at free cadence.

Information about respiratory frequency and oxygen consumption $\left(\dot{V} \mathrm{O}_{2}\right)$ were obtained by an open-circuit sampling system (Oxycon Pro, Jaeger-Viasys Healthcare, Hoechberg, Germany). The metabolic cart was calibrated with a known gas mixture (16\% oxygen, $\mathrm{O}_{2}$, and $5 \%$ carbon dioxide, $\mathrm{CO}_{2}$ ) and volume prior to the first test each day as recommended by the company. Both respiratory frequency and $\dot{V} \mathrm{O}_{2}$ data were interpolated at $4 \mathrm{~Hz}$ and low-pass filtered with a cut-off frequency of $0.01 \mathrm{~Hz}$ to obtain $f_{\mathrm{R}}(n)$ and $d_{\mathrm{VO}_{2}}(n)$ series, respectively. Five different intervals dependant on $d_{\dot{\mathrm{vo}}_{2}}(n)$ are defined: resting phase $\left(I_{\mathrm{B}}\right)$, increments up to $60 \%, 80 \%$ and $100 \%$ of the consumed oxygen $\left(I_{60}, I_{80}, I_{100}\right)$ and recovery phase $\left(I_{\mathrm{R}}\right)$.

In addition to the ECG recording, RR intervals were recorded beat-to-beat using an HR monitor (RS800, Polar Electro Oy, Kempele, Finland) which uses a sampling frequency of $1000 \mathrm{~Hz}$ for the ECG signal. RR intervals from the ECG $\left(\mathrm{RR}_{\mathrm{ECG}}\right)$ were obtained as the difference of each consecutive beat occurrences. RR intervals from Polar $\left(\mathrm{RR}_{\mathrm{POL}}\right)$ were directly obtained from the device. The delay between $R_{\mathrm{ECG}}$ and $\mathrm{RR}_{\mathrm{POL}}$ was estimated as that lag which maximizes their cross correlation. Subsequently, the two series were synchronized by correcting this delay.

\subsection{Data analysis}

The instantaneous heart rate signal, $d_{\mathrm{HR}}(n)$, is derived from both RR intervals series, following a method based on the TVIPFM model, and sampled at $4 \mathrm{~Hz}$. This signal is high-pass filtered to remove the mean heart rate $d_{\mathrm{HRM}}(n)$ (very low frequency components) and it is also corrected by it: $m(n)=\left(d_{\mathrm{HR}}(n)-d_{\mathrm{HRM}}(n)\right) / d_{\mathrm{HRM}}(n)$ [8].

The smoothed pseudo Wigner-Ville distribution (SPWVD) was applied to $m(n)$ to estimate the time-varying spectral properties of both HRV signals [9], with the same parameters used in [10]. The instantaneous power in the low and high frequency band, $P_{\mathrm{LF}}(n)$ and $P_{\mathrm{LF}}(n)$ respectively, was extracted throughout the entire exercise test. Low frequency band ranged from 0.04 to $0.15 \mathrm{~Hz}$. The high frequency band was centered on $f_{\mathrm{R}}(n)$ with a bandwidth of $0.25 \mathrm{~Hz}$. The lower limit of the HF band was never below $0.15 \mathrm{~Hz}$, and the upper limit was never above the half of mean heart rate [10]. Figure 1 shows an example of $P_{\mathrm{HF}}(n)$ from ECG and Polar during the resting phase and exercise phase $\left(I_{60}\right.$ to $\left.I_{100}\right)$. It can be seen that both powers are very similar at rest, but the differences become more evident during exercise.
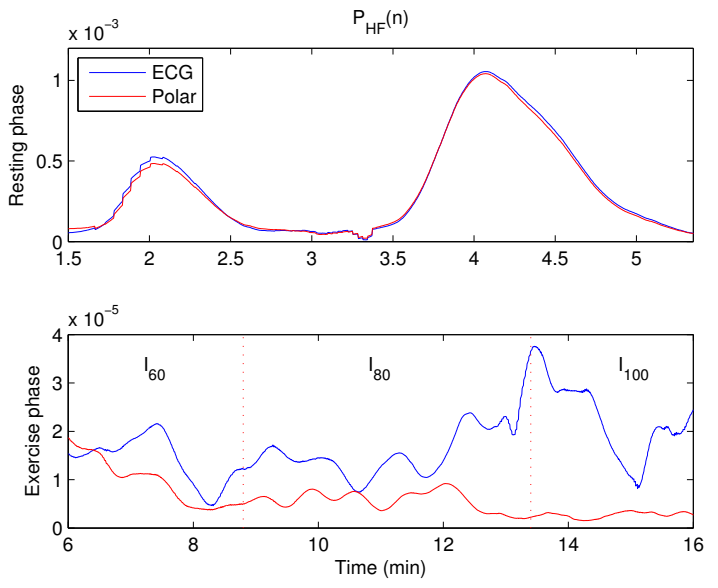

Figure 1. Example of $P_{\mathrm{HF}}(n)$ obtained from ECG and Polar during rest and exercise phase. Intervals $I_{60}, I_{80}$ and $I_{100}$ represents $0-60 \%, 60-80 \%$ and $80-100 \%$ of $d_{\dot{\mathrm{v}}_{2}}(n)$, respectively.

CC components are related to the cadence frequency, $f_{\mathrm{c}}(n)$, which is fixed at 80rpm. Since the intrinsic sampling frequency of HRV is the HR, when $f_{\mathrm{C}}(n)$ exceeds half the mean HR, aliasing occurs and aliased components appear in the visible part of the spectrum [3]. There are two main aliased components (denoted AF1 and AF2) and their powers are denoted as:

- $P_{\mathrm{AFI}}(n)$ : in a band centered at $d_{\mathrm{HRM}}(n)-f_{\mathrm{C}}(n)$ with a bandwidth of $0.125 \mathrm{~Hz}$.

- $P_{\mathrm{AF} 2}(n)$ : in a band centered at $-d_{\mathrm{HRM}}(n)+2 \cdot f_{\mathrm{C}}(n)$ with a bandwidth of $0.125 \mathrm{~Hz}$.

Figure 2 shows an example of a time-frequency map showing some of the spectral components of HRV, as well as an overlapping between $\mathrm{HF}$ and the aliased components. 


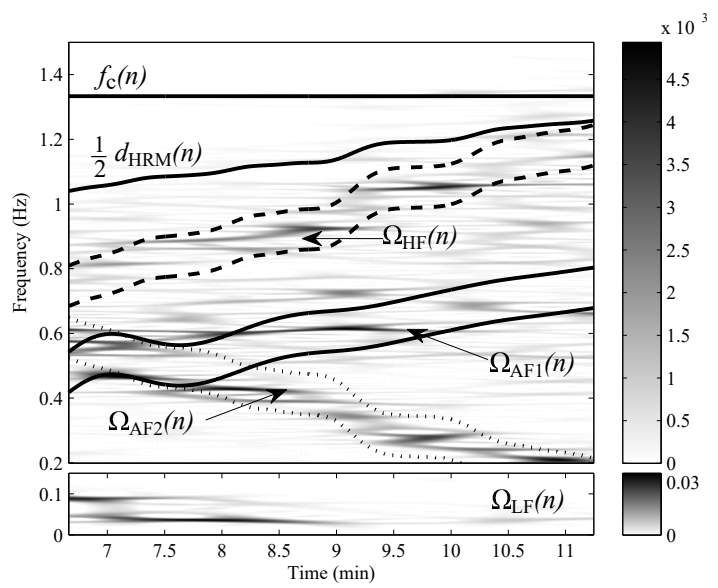

Figure 2. Example of a time-frequency map showing some of the spectral components of $\mathrm{HRV}$ (HF, CF, AF1 and AF2) during the exercise phase.

When components related to cardiolocomotor coupling overlap with HF band, it makes difficult to interpret it as a measure of respiratory sinus arrhythmia. A method was proposed in [11] and expanded in [12] to correct the powers which takes into account the percentage of overlap between the spectral bands and the relative power prior the overlapped area.

Due to the large changes of the total power for all bands, each instantaneous power is normalized by the instantaneous total power, which is defined as:

$$
P_{\mathrm{TOT}}(n)=P_{\mathrm{LF}}(n)+P_{\mathrm{HF}}(n)+P_{\mathrm{CC}}(n)
$$

with $P_{\mathrm{CC}}(n)$ being the sum of all components related to cardiolocomotor coupling, and both HF and CC components being corrected in the overlapped areas. Then, the studied power parameters are:

$$
\bar{P}_{\mathcal{A}}^{\mathrm{I}}=\frac{1}{N_{\mathrm{I}}} \sum_{n \in I} \frac{\hat{P}_{\mathcal{A}}(n)}{P_{\text {TOT }}(n)}, \quad I \in\left\{I_{\mathrm{B}}, I_{60}, I_{80}, I_{100}, I_{\mathrm{R}}\right\}
$$

Where $N_{\mathrm{I}}$ denotes the length of the interval $I$, and the subindex $\mathcal{A}$ indicates the chosen spectral component. Note that $\bar{P}_{\text {CC }}^{\mathrm{I}_{B}}$ is an exception and is not defined, since at rest there is no power related to cardiolocomotor coupling.

\subsection{Statistical analysis}

Parameters $\bar{P}_{\mathrm{LF}}^{\mathrm{I}}, \bar{P}_{\mathrm{HF}}^{\mathrm{I}}$ and $\bar{P}_{\mathrm{CC}}^{\mathrm{I}}$ were obtained for every interval. A Kolmogorov test showed that they did not follow a normal distribution. Therefore, a paired Wilcoxon test was applied for every parameter to study the differences Polar and ECG measurements to see if they show the same
ANS changes in the different intervals. The difference is considered to be significantly different from zero when $\mathrm{p}$ $<0.05$. Moreover, a Friedman test is applied for every parameter within the same type of measurements, followed by post-hoc comparisons to study the changes through the test.

\section{Results}

Figure 3 shows parameters $\bar{P}_{\mathrm{LF}}^{\mathrm{I}}, \bar{P}_{\mathrm{HF}}^{\mathrm{I}}$ and $\bar{P}_{\mathrm{CC}}^{\mathrm{I}}$ in the different intervals from the Polar and ECG measurements. LF power is always higher in Polar measurements, while HF power is always lower with respect to the ECG measurements, but no significant differences were found between ECG and Polar measurements.
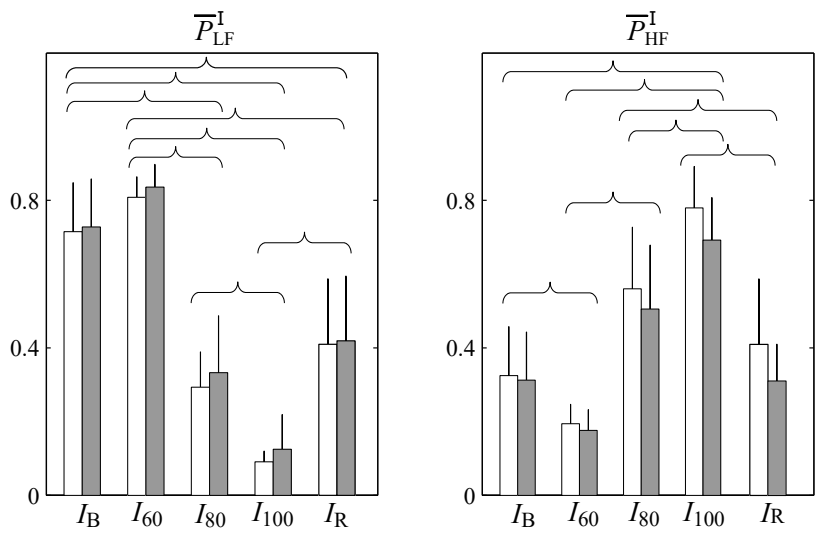

$\bar{P}_{\mathrm{CC}}^{\mathrm{I}}$

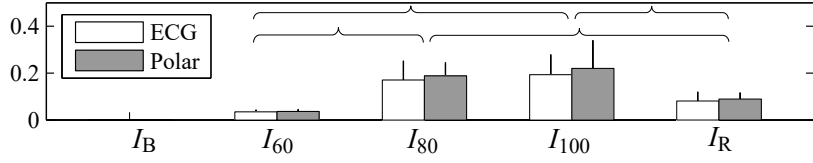

Figure 3. Median and MAD of $\bar{P}_{\mathrm{LF}}^{\mathrm{I}}, \bar{P}_{\mathrm{HF}}^{\mathrm{I}}$ and $\bar{P}_{\mathrm{HF}}^{\mathrm{I}}$ in intervals $I_{\mathrm{R}}, I_{60}, I_{80}$ and $I_{100}$ for the ECG and Polar measurements. Brackets denote significant differences ( $\mathrm{p}$ value $<$ $0.05)$.

Regarding the trend of the HRV measurements, $\bar{P}_{\mathrm{LF}}^{\mathrm{I}}$ increases at the beginning of the exercise (although it is not significant in this test), then it decreases again when the exercise load gets more intense, and then it increases in the recovery phase. All pair of intervals, with the exception of the pairs $I_{\mathrm{B}}$ and $I_{60}$, and $I_{80}$ and $I_{\mathrm{R}}$, present significant differences. $\bar{P}_{\mathrm{HF}}^{1}$ gets significantly reduced at $I_{60}$, then it increases and it decreases again in the recovery phase. $I_{\mathrm{B}}$ is significantly different to all intervals except $I_{80}$ and $I_{\mathrm{R}}$; $I_{60}$ is significantly different to all intervals except $I_{\mathrm{R}}$; and $I_{100}$ is significantly different to all intervals. There is a significative increase from $\mathrm{I}_{60}$ to $\mathrm{I}_{80}$ in $\bar{P}_{\mathrm{CC}}^{\mathrm{I}}$, and a significative decrease from $I_{100}$ to $I_{\mathrm{R}}$. 


\section{Discussion}

This study has shown that, besides the reported low values of reliability and agreement coefficients for HF power during high intensities of exercise in previous works, no significant differences were found in LF, HF and CC components when calculating power parameters as the mean value in the intervals of interest and correcting the $\mathrm{CC}$ components, regardless the intensity of exercise.

Both Polar and ECG measurements showed an increase in normalized LF power in low intensities, a significant decrease in medium to high intensities of exercise and then a significant increase in the recovery phase; with an opposite behaviour in the case of normalized HF. This behaviour supports the findings in other works, where it is shown that at the beginning of the exercise there are both a parasympathetic withdrawal and an augmentes sympathetic activity; but later on, during high intensities, HF increases due to the mechanical effect of breathing. There is also a greater cardiolocomotor power near the peak of exercise: CC components reached about $20 \%$ of the total power in the last exercise intervals and about $10 \%$ in the recovery phase.

This suggests that the same conclusions regarding the ANS response to exercise can be derived analyzing HRV derived from RR series provided by Polar than from RR series obtained from the ECG.

\section{Conclusion}

A Polar RS800 device was validated in 23 healthy male volunteers during an exercise test. A high resolution ECG was simultaneously recorded to extract the RR intervals and use them as a reference. A time-frequency spectral analysis was performed to extract the mean HR and the power of LF and HF components, the latter centered on the respiratory frequency. Although the performance of the HF measurements from the Polar device had been reported to decrease as the level of the exercise increases, with reliability and agreement coefficients around 0.5 , the total power in the HF band in the analyzed interval was found to be not significantly different from the ECG measurement.

\section{Acknowledgements}

This work has been supported by Centro de Iinvestigación Biomédica en Red - Bioingeniería, Biomateriales y Nanomedicina (CIBER-BBN) through Instituto de Salud Carlos III, by MINECO and FEDER under project TIN2014-53567-R and DPI2016-75458-R, and by Aragón Gobernment (Spain) and European Social Fund through Grupo Consolidado Biomedical Signal Interpretation and Computational Simulation (BSICoS), Ref: T96. The computation of some parts of this work was performed at the High Performance Computing platform of the NANBIOSIS
ICTS, CIBER-BBN and Aragón Institute of Engineering Research (I3A), Zaragoza, Spain.

\section{References}

[1] Kumar M, Weippert M, Vilbrandt R, Kreuzfeld S, Stoll R. Fuzzy evaluation of heart rate signals for mental stress assessment. IEEE Trans Fuzzy Syst 2007;15:791-808.

[2] Kaber DB, Perry CM, Segall N, Sheik-Nainar MA. Workload state classiffcation with automation during simulated air traffic control. Int J Aviat Psychol 2007;17:371-390.

[3] Turner SE, Eastwood PR, Cecins NM, Hillman DR, Jenkins SC. Physiologic responses to incremental and self-paced exercise in COPD: a comparison of three tests. Chest 2004; 126:766-773.

[4] Gamelin FX, Baquet G, Berthoin S, Bosquet L. Validity of the polar S810 to measure R-R intervals in children. Int J Sports Med 2008;29(2):134-138.

[5] Giles D, Draper N, Neil W. Validity of the Polar V800 heart rate monitor to measure RR intervals at rest. Eur J Appl Physiol 2016;116:563-571.

[6] Bouillod A, Cassirame J, Bousson JM, Sagawa Y, Tordi N. Accuracy of the Suunto system for heart rate variability analysis during a tilt-test. Rev Bras Cineantropom Desempenho 2015;17:410-417.

[7] Hernando D, Garatachea N, Almeida R, Casajús J, Bailón R. Validation of heart rate monitor polar RS800 for heart rate variability analysis during exercise. Journal of Strength and Conditioning Research 2017;

[8] Bailón R, Laouini G, Grao C, Orini M, Laguna P, Meste O. The integral pulse frequency modulation with time-varying threshold: application to heart rate variability analysis during exercise stress testing. IEEE Trans Biomed Eng 2011; 58 (3):642-652.

[9] Martin W, Flandrin P. Wigner-Ville spectral analysis of nonstationary processes. IEEE Trans Acoust Speech Signal Process 1985;33:1461-1470.

[10] Bailón R, Garatachea N, I. de la Iglesia, JA. Casajús, Laguna $\mathrm{P}$. Influence of running stride frequency in heart rate variability analysis during treadmill exercise testing. IEEE Trans Biomed Eng 2013;60(7):1796-1805.

[11] Hernando A, Hernando D, Garatachea N, Casajús J, Bailón R. Attenuation of the influence of cardiolocomotor coupling in heart rate variability interpretation during exercise test. In 37nd Annual International Conference of the IEEE EMBS. 2015; 1508-1511.

[12] Hernando D, Hernando A, Casajús J, Laguna P, Garatachea N, Bailón R. Methodological framework for heart rate variability analysis during exercise: application to running and cycling stress testing. Med Biol Eng Comput 2017;DOI 10.1007/s11517-017-1724-9.

Address for correspondence:

David Hernando (dhernand@unizar.es). Mariano Esquillor s/n, Ed. I+D+i, L4.0.04, 50018, Zaragoza (Spain). 